\title{
Visit efsumb.org to view the Euroson School programmes
}

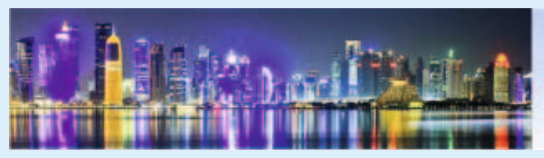

5th Qatar Musculoskeletal Ultrasound Course
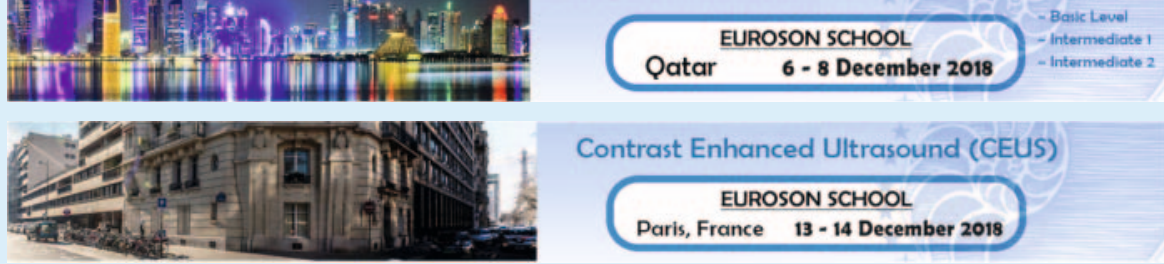

Contrast Enhanced Ultrasound (CEUS)

EUROSON SCHOOL

Paris, France $13-14$ December 2018

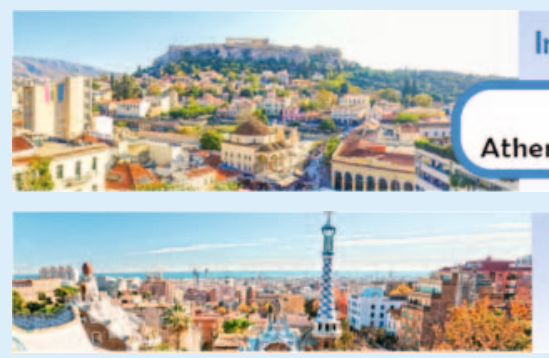

Interventional Ultrasound \& Elastography

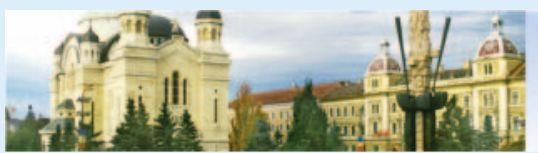

EUROSON SCHOOL

Athens, Greece 18 - 19 January 2019

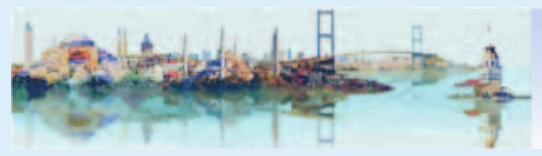

Elastography, Current US Techniques \& Interventional US

EUROSON SCHOOL
Istanbul 28 - 30 March 2019

Looking back:

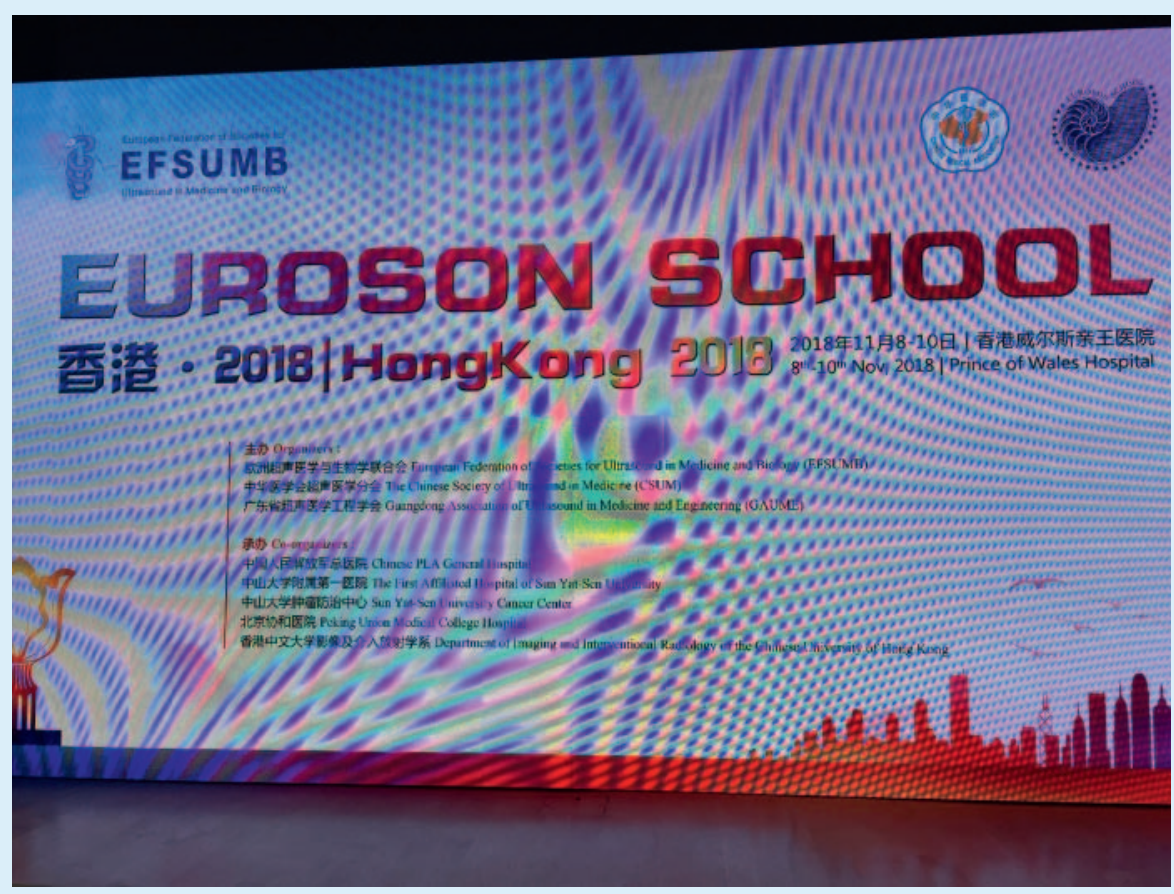

The CEUS School held in Hong Kong in November was a huge success with 200 participants. Plans are already underway for a school in 2019. 


\section{Looking forward:}

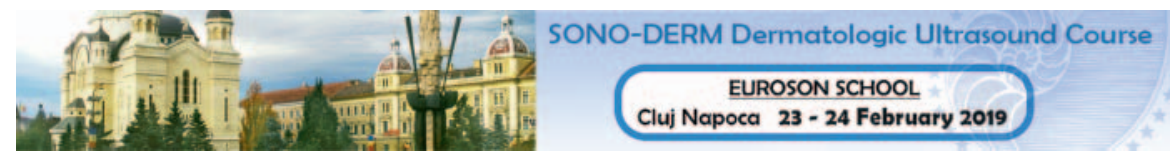

\section{Euroson School Cluj Napoca February 2019}

The ulrasonographic assessment represents a new method for the non-invasive evaluation in dermatology. Usually in dermatology the diagnosis is based on physical examination, palpation, history, dermoscopy and histology. Nowadays the modern sonographic equipments allow a multimodal and integrative assessment of skin pathology that includes valuable parameters related to morphology, blood supply, elasticity, volume, etc.

There is an increased interest for the preoperative assessment and postoperative follow-up of skin tumours. The preoperative information about the size and the thickness of the tumour is of practical relevance especially for melanomas and carcinomas regarding the margins of excision, sentinel node, local recurrences. The sonographic parameters can be used for monitoring the course and the response to treatment of different chronic inflammatory disorders like morphea, psoriasis, dermatomyositis. The high frequency ultrasound using transducers up to $30 \mathrm{MHz}$ has widened the clinical applications to the assessment of wound healing, scars, efficacy of different cosmetic products, side effects after cosmetic procedures etc.

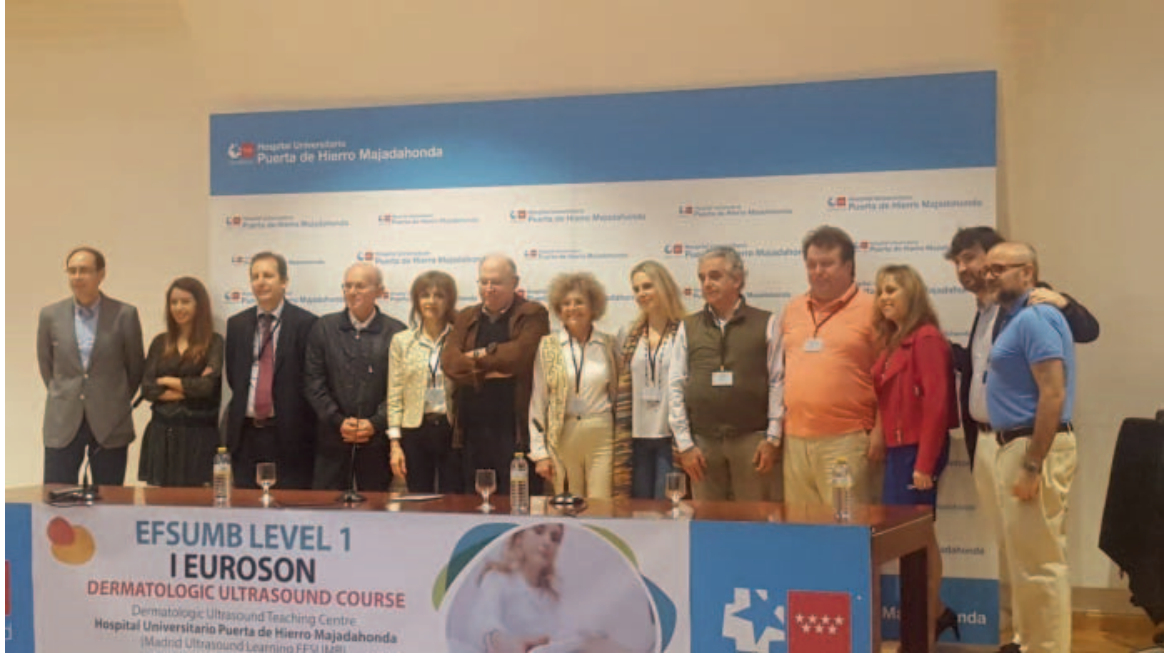

Contribution from Prof Dr Maria Crispn and Prof Dr Radu Badea, University of Medicine and Pharmacy, „Iulia Hatieganu“ Cluj Napoca. Photo of some of the authors of the EFSUMB Dermatological Guidelines (work in progress) who met in Madrid in 2019.

Even histology remains the "gold standard for diagnosis", researchers are trying to find skin ultrasound features as close as possible to the histological ones. Related to the literature data and the conferences held in Orlando (2017), Madrid and Barcelona (2018), sonography can be successfully implemented in dermatology as a complementary, non-invasive method that can identify several parameters with histological precision, contributing to the diagnosis, prognosis, and therapeutical approach of skin pathology. 